\title{
MHD Natural Convective Heat Generation/Absorbing and Radiating Fluid Past a Vertical Plate Embedded in Porous Medium - an Exact Solution
}

\author{
P. Chandra Reddy ${ }^{1 *}$, M. C. Raju ${ }^{2}$, G. S. S. Raju² \\ ${ }^{1}$ Department of Mathematics, Annamacharya Institute of Technology and Sciences \\ (Autonomous), Rajampet-516126, A.P., India. \\ ${ }^{2}$ Department of Mathematics, JNTUA College of Engineering, Pulivendula, A.P., India. \\ E-mails: chandramsc01@gmail.com; mcrmaths@yahoo.co.in \\ *corresponding author
}

\begin{abstract}
The present work reveals the examination of the characteristics of MHD free convective radiating fluid past a permeable plate with the occurrence of thermal transmission and heat source/sink along with changeable concentration and temperature. An exact solution has been employed by usual Laplace transform technique. The effects of diverse parameters on flow velocity, hotness and concentration are discussed through graphical representations and tables. With the incidence of heat source, the fluid velocity and temperature increases whereas the concentration decreases. The velocity and temperature falls down in the incidence of heat drop. Escalating values of Soret number serves to enhance the velocity and species concentration but an opposite nature is found with Schmidt number. The current study is well supported by the verification of previously published results.
\end{abstract}

Keywords: Free convection, Thermal radiation, Heat source/sink, Porous plate and Thermal diffusion.

\section{Introduction}

Magnetohydrodynamic free convective fluid flows with special geometries implanted in porous media have many engineering, industrial and geophysical applications. Some of them are utilized in geothermal reservoirs, drying process and thermal filling. A renewed interest was carried out in studying magnetohydrodynamic (MHD) flow with double diffusion in porous media under flow control as well as on the presentation of many systems using electrically conducting fluids. This type of fluid flows with thermal diffusion, heat source/sink, chemical reaction and thermal radiation has attracted the interest of many researchers. As a result plenty of research articles have been employed. Raju et al. (2011) analyzed MHD flow linking heated tending plates. Kim (2001) established unsteady magnetohydrodynamic convection flow of polar fluids through a vertical moving plate. A similar study was done by Seth et al. (2015). Hayat and Qasim (2010) as well as Vajravelu and Kumar (2004) employed systematic and statistical solutions of coupled nonlinear system arising in three-dimensional rotating flow. Murthy et al. (2011) analyzed this over nondarcy porous media. Soret effect on non-Newtonian liquid was studied by Narayana et al. (2013). 
Sandeep et al. (2012) discussed the conseuences of emission and compound result on transient convective flow. Kandasamy et al. (2005) and Makinde et al. (2013) examined buoyancy effects on magnetohydrodynamic stagnation point flow of a nanofluid. Chamkha et al. (2011) analyzed unsteady MHD flow along a straight up cylinder. Chamkha et al. (2011) also studied the characteristics of MHD convection flow with the effects of Hall current, thermal emission and chemical reaction. Hossain et al. (2001) and Reddy et al. (2013) analyzed the similar aspects by changing boundary conditions. Further Reddy et al. (2015), Srinivasacharya and RamReddy (2013) examined cross-transmission impact on the flows in non-darcy porous medium. Muthucumarswamy and Kumar (2004) analyzed and reported double transfer impacts on flow under thermic emission. Ahmed and Kalita (2013) analyzed theoretically the magnetohydrodynamic transitory flow. Seddeek (2000) and Sharma et al. (2011) considered and reported the effect of rays on temperature distribution over three-dimensional Couette stream with heat source/sink. Raptis (1986) established the physical properties of the fluid flow implementing magnetic field. Das et al. (2012) analyzed rays effects on fluid flow under the existence of Newtonian heating. Narahari and Nayan (2011) investigated the properties of free convection flow with heating radiation and species dispersion.

On the other hand many researchers contributed in studying the characteristics of magnetohydrodynamic free convective fluid flow through porous medium under the effects of dissimilar physical parameters by considering variable temperature and concentration. Muthucumaraswamy et al. (2009) analyzed unsteady flow through a movable immeasurable vertical plate. Uwanta and Sarki (2012) examined the physical properties of heat and mass transfer with varying temperature and exponential mass diffusion. Suneetha et al. (2008) discussed heating radiation impact on MHD natural convection fluid flow through an impetuously started upright plate with inconsistent temperature and concentration. Keeping in mind the above studies we made an attempt to analyze the characteristics of MHD free convective heat generating and radiating fluid flow past a porous plate in the presence of thermal diffusion by considering variable temperature and concentration. We have extended the study of Pattnaik and Biswal (2015) which gives analytical solution of MHD natural convective flow through porous media with time dependent temperature and concentration. The novelty of this study is the consideration of thermal radiation and Soret effects along with variable temperature and concentration. The existence of Soret effect and thermal radiation are considered based on their significance in continuous research in chemical industries and water purification process.

Further, we have gone through some recent studies related to present analysis and followed the findings. Awais et al. (2016) analyzed the slip effects on a magneto-hydrodynamic flow of nanofluid. Das et al. (2015) considered and studied the nature of Casson flows introducing Newtonian heating. Hayat et al. (2013) examined the properties miscellaneous MHD convection flow of thyrotrophic fluid with thermophoresis and Joule heating. Shibdas Dholey (2016) compared the flow of two non-Newtonian fluids over a superior flat shell of parabolic revolution. Azad Hussain and Anwar Ullah (2016) examined the nature of extreme flows of a Walter's B fluid caused by a stretchable cylinder by way of temperature reliant viscosity.

\section{Nomenclature}

$\mathrm{u}^{*} \quad$ Velocity component along the $\mathrm{x}^{*}$-axis

$\mathrm{T}^{*} \quad$ Fluid temperature

$T_{w}^{*} \quad$ Temperature at the plate

u non-dimensional velocity

$C^{*} \quad$ Species concentration

$T_{\infty}$ Temperature at infinity

$C_{\infty}^{*} \quad$ Concentration at infinity

$t^{*} \quad$ Time

$g \quad$ Acceleration due to gravity

$\theta \quad$ Non-dimensional temperature

$C$ non-dimensional concentration

$C_{w}^{*}$ Concentration at the plate

$t \quad$ Non-dimensional time

$\mathrm{B}_{0} \quad$ Magnetic field component along $\mathrm{y}^{*}$ 


$\begin{array}{llcl}K_{p}^{*} & \text { Permeability of the medium } & K & \text { porosity parameter } \\ C_{p} & \text { Specific heat at constant pressure } & \mathrm{k} & \text { Thermal conductivity } \\ \beta & \text { Volumetric coefficient of expansion for } & \beta^{*} & \text { Volumetric coefficient of expansion } \\ & \text { heat transfer } & & \text { for mass transfer } \\ \mathrm{Q}_{0} & \text { Volumetric heat generation / absorption } & \mathrm{Q} & \text { heat source parameter } \\ D & \text { Molecular diffusivity } & G c & \text { modified Grashof number } \\ G r & \text { Grashof number for heat transfer } & M & \text { magnetic parameter } \\ P r & \text { Prandtl number } & \mathrm{R} & \text { Radiation parameter } \\ D_{1} & \text { Thermal diffusivity } & \text { So } & \text { Soret number } \\ S c & \text { Schmidt number } & u_{0} & \text { Characteristic velocity } \\ N u & \text { Nusselt number } & S h & \text { Sherwood number } \\ v & \text { Kinematic coefficient of viscosity } & \rho & \text { Density of the fluid } \\ \sigma & \text { Electrical conductivity of the fluid } & \tau & \text { Skin friction }\end{array}$

\section{Formulation of the problem}

The unsteady MHD free convective heat generating/absorbing and radiating fluid past a porous plate in the presence of thermal diffusion with inconsistent temperature and concentration has been considered. The physical form and coordinate structure of the fluid flow is accessible in Fig. 1. The flow is assumed to be in $x^{*}$-direction which is taken along the vertical plate in the uphill direction. The $\mathrm{y}^{*}$-axis is taken be normal to the plate. Initially, it is assumed that both the fluid and the plate are at rest and maintained at same temperature and concentration $T_{\infty}^{*}$ and $C_{\infty}^{*}$ respectively. At any time $t^{*}>0$ the temperature and concentration of the plate $y^{*}=0$ are raised to $T_{\infty}^{*}+\left(T_{w}^{*}-T_{\infty}^{*}\right) \mathrm{e}^{a^{* *} t^{*}}$ and $C_{\infty}^{*}+\left(C_{w}^{*}-C_{\infty}^{*}\right) \mathrm{e}^{a^{* *} t^{*}}$ with time $\mathrm{t}$ and thereafter remains constant and that of $y^{*} \rightarrow \infty$ is lowered to $T_{\infty}^{*}$ and $C_{\infty}^{*}$ respectively. A transverse magnetic field of standardized potency is assumed to be applied at a 90 degree angle to the plate. The induced magnetic field and viscous dissipation is taken to be insignificant as the magnetic Reynolds number is assumed as extremely little for this flow.

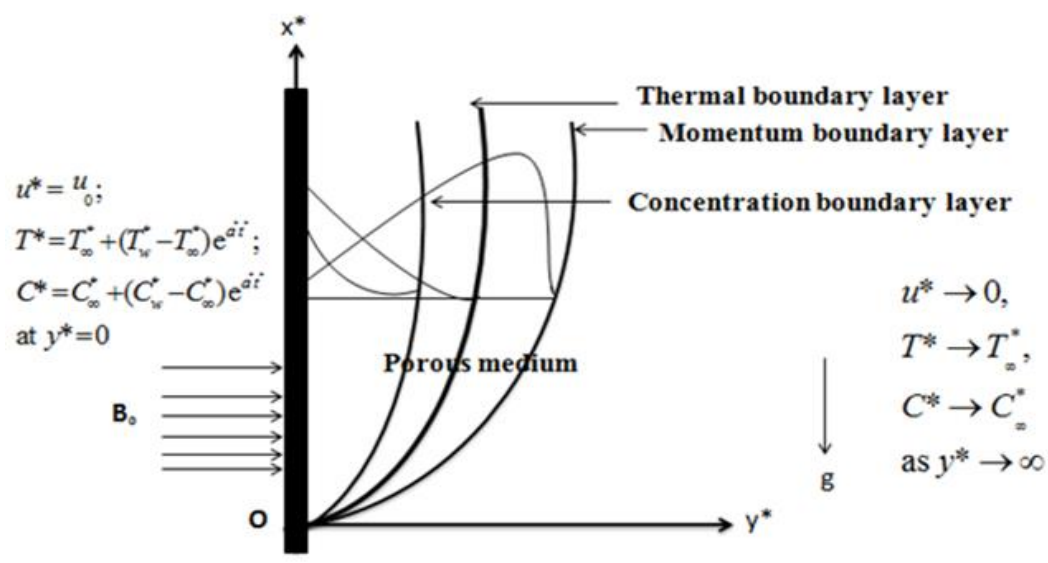

Fig. 1. Flow geometry and coordinate system 
The Soret effect is measured and studied whereas Dufour effect is negligible. The Hall effect of magnetohydrodynamics and magnetic dissipation (Joule heating of the fluid) are abandoned. Based on the above assumption with usual Boussineq's approximation, the governing equations and related boundary conditions of the problem are given by

Momentum equation:

$$
\frac{\partial u^{*}}{\partial t^{*}}=v \frac{\partial^{2} u^{*}}{\partial y^{* 2}}+g \beta^{*}\left(C^{*}-C_{\infty}^{*}\right)+g \beta\left(T^{*}-T_{\infty}^{*}\right)-\frac{\sigma B_{0}^{2}}{\rho} u^{*}-\frac{v}{K_{p}^{*}} u^{*}
$$

Energy equation:

$$
\rho C_{p} \frac{\partial T^{*}}{\partial t^{*}}=k \frac{\partial^{2} T^{*}}{\partial y^{* 2}}+q_{0}\left(T^{*}-T_{\infty}^{*}\right)-\frac{\partial q_{r}^{*}}{\partial y^{*}}
$$

Concentration equation:

$$
\frac{\partial C^{*}}{\partial t^{*}}=D \frac{\partial^{2} C^{*}}{\partial y^{*^{2}}}+D_{1} \frac{\partial^{2} T^{*}}{\partial y^{*^{2}}}
$$

Initial and Boundary conditions:

$$
\begin{gathered}
u^{*}=0, \mathrm{C}^{*}=C_{\infty}^{*}, T^{*}=T_{\infty}^{*} \quad \text { for all } y^{*}, t^{*} \leq 0 \\
t^{*}>0: u^{*}=u_{0}, \mathrm{C}^{*}=C_{\infty}^{*}+\left(C_{w}^{*}-C_{\infty}^{*}\right) e^{a^{*} t^{*}}, \mathrm{~T}^{*}=T_{\infty}^{*}+\left(T_{w}^{*}-T_{\infty}^{*}\right) e^{a^{*} t^{*}} \text { at } y^{*}=0 \\
u^{*} \rightarrow 0, \quad \mathrm{C}^{*} \rightarrow C_{\infty}^{*}, T^{*} \rightarrow T_{\infty}^{*} \quad \text { as } y^{*} \rightarrow \infty
\end{gathered}
$$

The confined radiant inclusion for the situation of an optically slight gray gas is collected and expressed (Cogley et al. and Raju et al.) as

$$
\frac{\partial q_{r}^{*}}{\partial y^{*}}=4 a^{\prime} \sigma^{*}\left(T_{\infty}^{*^{4}}-T^{*^{4}}\right)
$$

where $\sigma^{*}$ and $a^{\prime}$ are the Stefan-Boltzmann constant and absorption mean co-efficient respectively. We assume that the variations contained by the flow are suitably little so that $T^{* 4}$ is reduced as a linear function in $T^{*}$ with the use of Taylor's series to enlarge $T^{*^{4}}$ with respect to free stream temperature $T_{\infty}^{* 4}$ and by avoiding the terms having higher order. The outcome will be in the following approximation: $T^{* 4} \cong 4 T_{\infty}^{* 3} T^{*}-3 T_{\infty}^{* 4}$. Then equation (2) takes the following form

$$
\rho C_{p} \frac{\partial T^{*}}{\partial t^{*}}=k \frac{\partial^{2} T^{*}}{\partial y^{*^{2}}}+q_{0}\left(T^{*}-T_{\infty}^{*}\right)-16 a^{\prime} \sigma^{*} T_{\infty}^{*^{3}}\left(T^{*}-T_{\infty}^{*}\right)
$$

The following non-dimensional quantities are introduced:

$$
u=\frac{u^{*}}{u_{0}}, \quad t=\frac{t^{*} u_{0}^{2}}{v}, \quad y=\frac{y^{*} u_{0}}{v}, \quad \theta=\frac{T^{*}-T_{\infty}^{*}}{T_{w}^{*}-T_{\infty}^{*}}, \quad \mathrm{C}=\frac{C^{*}-C_{\infty}^{*}}{C_{w}^{*}-C_{\infty}^{*}}
$$

After introducing the non-dimensional quantities into the equations (1), (3) and (6), they condense to the following form: 


$$
\begin{gathered}
\frac{\partial u}{\partial t}=\frac{\partial^{2} u}{\partial y^{2}}+G r \theta+G c C-M u-\frac{1}{K} u \\
\operatorname{Pr} \frac{\partial \theta}{\partial t}=\frac{\partial^{2} \theta}{\partial y^{2}}+Q \theta-R \theta \\
\frac{\partial C}{\partial t}=\frac{1}{S c} \frac{\partial^{2} C}{\partial y^{2}}+S o \frac{\partial^{2} \theta}{\partial y^{2}}
\end{gathered}
$$

where

$$
\begin{aligned}
& \operatorname{Pr}=\frac{\mu C_{p}}{k} \text { (Prandtl number) } \\
& G r=\frac{v g \beta\left(T_{w}^{*}-T_{\infty}^{*}\right)}{u_{0}^{3}} \text { (Grashof number) } \\
& G c=\frac{v g \beta^{*}\left(C_{w}^{*}-C_{\infty}^{*}\right)}{u_{0}^{3}} \quad(\text { Solutal Grashof number }) \\
& M=\frac{\sigma B_{0}^{2} v}{\rho u_{0}^{2}} \quad \text { (Magnetic parameter) } \\
& K=\frac{K_{p}^{*} u_{0}^{2}}{v^{2}} \quad \text { (Porosity parameter) } \\
& R=\left(\frac{16 a^{\prime} \sigma^{*} v^{2} T_{\infty}^{*^{3}}}{k u_{2}^{2}}\right) \quad \text { (Radiation parameter) } \\
& S c=\frac{v}{D} \quad \text { (Schmidt number) } \\
& Q=\frac{q_{0} v^{2}}{k u_{0}^{2}} \quad \text { (Heat generation parameter) } \\
& S o=\frac{D_{1}}{v} \frac{\left(T_{w}^{*}-T_{\infty}^{*}\right)}{\left(C_{w}^{*}-C_{\infty}^{*}\right)} \quad \text { (Soret number) }
\end{aligned}
$$

The corresponding initial and boundary conditions are as follows:

$$
\begin{aligned}
& u=0, \quad \theta=0, \quad \mathrm{C}=0 \quad \text { for all } y, \mathrm{t} \leq 0 \\
& t>0: u=1, \quad \theta=e^{a t}, \quad \mathrm{C}=e^{a t} \quad \text { at } y=0 \\
& u \rightarrow 0, \theta \rightarrow 0, \mathrm{C} \rightarrow 0 \quad \text { as } y \rightarrow \infty
\end{aligned}
$$




\section{Solution of the problem}

The governing equations (8) - (10) which are non-linear partial differential equations along with the boundary conditions (11) are solved by applying Laplace transform technique. The expressions $u(y, t), \theta(y, t), C(y, t)$ are the functions of $\mathrm{y}$ and $\mathrm{t}$. Applying Laplace transforms to the equations $(8)-(10)$ they reduced to the functions of $y$ and $s$ and by the use of boundary conditions (11) we get the following expressions:

$$
\begin{aligned}
& \bar{u}(y, s)=\frac{1}{s} e^{-y \sqrt{s+A_{1}}}-A_{7} \frac{1}{s-a} e^{-y \sqrt{s+A_{1}}}+A_{7} \frac{1}{s-b_{8}} e^{-y \sqrt{s+A_{1}}}- \\
& A_{8} \frac{1}{s-a} e^{-y \sqrt{s+A_{1}}}+A_{8} \frac{1}{s-b_{9}} e^{-y \sqrt{s+A_{1}}} \\
& -A_{9} \frac{1}{s-b_{4}} e^{-y \sqrt{s+A_{1}}}+A_{9} \frac{1}{s-b_{9}} e^{-y \sqrt{s+A_{1}}}-A_{10} \frac{1}{s-b_{4}} e^{-y \sqrt{s+A_{1}}}+ \\
& A_{10} \frac{1}{s-b_{8}} e^{-y \sqrt{s+A_{1}}} \\
& +A_{7} \frac{1}{s-a} e^{-y \sqrt{\operatorname{Pr}\left(s+b_{1}\right)}}-A_{7} \frac{1}{s-b_{8}} e^{-y \sqrt{\operatorname{Pr}\left(s+b_{1}\right)}}+A_{8} \frac{1}{s-a} e^{-y \sqrt{S c} \sqrt{s}}- \\
& A_{8} \frac{1}{s-b_{9}} e^{-y \sqrt{S c} \sqrt{s}} \\
& +A_{9} \frac{1}{s-b_{4}} e^{-y \sqrt{s c} \sqrt{s}}-A_{9} \frac{1}{s-b_{9}} e^{-y \sqrt{s c} \sqrt{s}}+A_{10} \frac{1}{s-b_{4}} e^{-y \sqrt{\operatorname{Pr}} \sqrt{s+b_{1}}}- \\
& A_{10} \frac{1}{s-b_{8}} e^{-y \sqrt{\operatorname{Pr}} \sqrt{s+b_{1}}} \\
& \bar{\theta}(y, s)=\frac{1}{s-a} e^{-y \sqrt{\operatorname{Pr}\left(s+b_{1}\right)}} \\
& \bar{C}(y, s)=\frac{1}{s-a} e^{-y \sqrt{S c} \sqrt{s}}+b_{6} \frac{1}{s-a} e^{-y \sqrt{s c} \sqrt{s}}+b_{7} \frac{1}{s-b_{4}} e^{-y \sqrt{S c} \sqrt{s}} \\
& -b_{6} \frac{1}{s-a} e^{-y \sqrt{\operatorname{Pr}} \sqrt{s+b_{1}}}-b_{7} \frac{1}{s-b_{4}} e^{-y \sqrt{\operatorname{Pr}} \sqrt{s+b_{1}}}
\end{aligned}
$$

By applying inverse Laplace transforms to the expressions in (12) - (14), the functions of y and $\mathrm{s}$ are transformed to the functions of $\mathrm{y}$ and $\mathrm{t}$. As a result, we get the solution of the governing equations (8) - (10) which involves all the physical parameters involved.

The accurate expressions for velocity, temperature and concentration are as follows:

$$
\begin{gathered}
u(y, t)=u_{11}(y, t)-A_{7} u_{12}(y, t)+A_{7} u_{13}(y, t)-A_{8} u_{12}(y, t)+A_{8} u_{14}(y, t)- \\
A_{9} u_{15}(y, t)+A_{9} u_{14}(y, t)-A_{10} u_{15}(y, t)+A_{10} u_{13}(y, t)+A_{7} u_{16}(y, t) \\
-A_{7} u_{17}(y, t)+A_{8} u_{18}(y, t)-A_{8} u_{19}(y, t)+A_{9} u_{20}(y, t)-A_{9} u_{19}(y, t)+ \\
A_{10} u_{21}(y, t)-A_{10} u_{17}(y, t) \\
\theta(y, t)=u_{16}(y, t) \\
C(y, t)=\left(1+b_{6}\right) u_{18}(y, t)+b_{7} u_{20}(y, t)-b_{6} u_{16}(y, t)-b_{7} u_{21}(y, t)
\end{gathered}
$$

Where 


$$
\begin{aligned}
& A_{1}=M+\frac{1}{K} \quad A_{2}=\frac{-G r}{\operatorname{Pr}-1} \quad A_{3}=\frac{-G c\left(1+b_{6}\right)}{S c-1} \\
& A_{4}=\frac{-G c b_{7}}{S c-1} \quad A_{5}=\frac{G c b_{6}}{\operatorname{Pr}-1} \quad A_{6}=\frac{G c b_{7}}{\operatorname{Pr}-1} \\
& A_{7}=\frac{A_{2}+A_{5}}{a-b_{8}} \quad A_{8}=\frac{A_{3}}{a-b_{9}} \quad A_{9}=\frac{A_{4}}{b_{4}-b_{9}} \\
& A_{10}=\frac{A_{6}}{b_{4}-b_{8}} \quad b_{1}=\frac{R-Q}{\operatorname{Pr}} \quad b_{2}=S c S o \\
& b_{3}=1-\frac{S c}{\operatorname{Pr}} \quad b_{4}=\frac{-b_{1}}{b_{3}} \quad b_{5}=\frac{b_{2}}{b_{3}} \\
& b_{6}=\frac{b_{5}\left(a+b_{1}\right)}{a-b_{4}} b_{7}=\frac{-b_{5}\left(b_{4}+b_{1}\right)}{a-b_{4}} \\
& b_{8}=\frac{A_{1}-\operatorname{Pr} b_{1}}{\operatorname{Pr}-1} \quad b_{9}=\frac{A_{1}}{S c-1} \\
& u_{11}(y, t)=\frac{1}{2}\left[e^{-y \sqrt{A_{1}}} \operatorname{erfc}\left(\frac{y}{2 \sqrt{t}}-\sqrt{A_{1} t}\right)+e^{y \sqrt{A_{1}}} \operatorname{erfc}\left(\frac{y}{2 \sqrt{t}}+\sqrt{A_{1} t}\right)\right] \\
& u_{12}(y, t)=\frac{e^{a t}}{2}\left[\begin{array}{r}
e^{-y \sqrt{a+A_{1}}} \operatorname{erfc}\left(\frac{y}{2 \sqrt{t}}-\sqrt{\left(a+A_{1}\right) t}\right)+ \\
e^{y \sqrt{a+A_{1}}} \operatorname{erfc}\left(\frac{y}{2 \sqrt{t}}+\sqrt{\left(a+A_{1}\right) t}\right)
\end{array}\right] \\
& u_{13}(y, t)=\frac{e^{b_{8} t}}{2}\left[\begin{array}{r}
e^{-y \sqrt{b_{8}+A_{1}}} \operatorname{erfc}\left(\frac{y}{2 \sqrt{t}}-\sqrt{\left(b_{8}+A_{1}\right) t}\right)+ \\
e^{y \sqrt{b_{8}+A_{1}}} \operatorname{erfc}\left(\frac{y}{2 \sqrt{t}}+\sqrt{\left(b_{8}+A_{1}\right) t}\right)
\end{array}\right] \\
& u_{14}(y, t)=\frac{e^{b_{9} t}}{2}\left[\begin{array}{r}
e^{-y \sqrt{b_{9}+A_{1}}} \operatorname{erfc}\left(\frac{y}{2 \sqrt{t}}-\sqrt{\left(b_{9}+A_{1}\right) t}\right)+ \\
e^{y \sqrt{b_{9}+A_{1}}} \operatorname{erfc}\left(\frac{y}{2 \sqrt{t}}+\sqrt{\left(b_{9}+A_{1}\right) t}\right)
\end{array}\right] \\
& u_{15}(y, t)=\frac{e^{b_{4} t}}{2}\left[\begin{array}{r}
e^{-y \sqrt{b_{4}+A_{1}}} \operatorname{erfc}\left(\frac{y}{2 \sqrt{t}}-\sqrt{\left(b_{4}+A_{1}\right) t}\right)+ \\
e^{y \sqrt{b_{4}+A_{1}}} \operatorname{erfc}\left(\frac{y}{2 \sqrt{t}}+\sqrt{\left(b_{4}+A_{1}\right) t}\right)
\end{array}\right]
\end{aligned}
$$




$$
\begin{aligned}
& u_{16}(y, t)=\frac{e^{a t}}{2}\left[\begin{array}{c}
e^{-y \sqrt{\operatorname{Pr}\left(a+b_{1}\right)}} \operatorname{erfc}\left(\frac{y \sqrt{\operatorname{Pr}}}{2 \sqrt{t}}-\sqrt{\left(a+b_{1}\right) t}\right)+ \\
e^{y \sqrt{\operatorname{Pr}\left(a+b_{1}\right)}} \operatorname{erfc}\left(\frac{y \sqrt{\operatorname{Pr}}}{2 \sqrt{t}}+\sqrt{\left(a+b_{1}\right) t}\right)
\end{array}\right] \\
& u_{17}(y, t)=\frac{e^{b_{8} t}}{2}\left[\begin{array}{c}
e^{-y \sqrt{\operatorname{Pr}\left(b_{8}+b_{1}\right)}} \operatorname{erfc}\left(\frac{y \sqrt{\operatorname{Pr}}}{2 \sqrt{t}}-\sqrt{\left(b_{8}+b_{1}\right) t}\right)+ \\
e^{y \sqrt{\operatorname{Pr}\left(b_{8}+b_{1}\right)}} \operatorname{erfc}\left(\frac{y \sqrt{\operatorname{Pr}}}{2 \sqrt{t}}+\sqrt{\left(b_{8}+b_{1}\right) t}\right)
\end{array}\right] \\
& u_{18}(y, t)=\frac{e^{a t}}{2}\left[\begin{array}{r}
e^{-y \sqrt{a S c}} \operatorname{erfc}\left(\frac{y \sqrt{S c}}{2 \sqrt{t}}-\sqrt{a t}\right)+ \\
e^{y \sqrt{a S c}} \operatorname{erfc}\left(\frac{y \sqrt{S c}}{2 \sqrt{t}}+\sqrt{a t}\right)
\end{array}\right] \\
& u_{19}(y, t)=\frac{e^{b_{9} t}}{2}\left[\begin{array}{r}
e^{-y \sqrt{b_{9} S c}} \operatorname{erfc}\left(\frac{y \sqrt{S c}}{2 \sqrt{t}}-\sqrt{b_{9} t}\right)+ \\
e^{y \sqrt{b_{9} S c}} \operatorname{erfc}\left(\frac{y \sqrt{S c}}{2 \sqrt{t}}+\sqrt{b_{9} t}\right)
\end{array}\right] \\
& u_{20}(y, t)=\frac{e^{b_{4} t}}{2}\left[\begin{array}{c}
e^{-y \sqrt{b_{4} S c}} \operatorname{erfc}\left(\frac{y \sqrt{S c}}{2 \sqrt{t}}-\sqrt{b_{4} t}\right)+ \\
e^{y \sqrt{b_{4} S c}} \operatorname{erfc}\left(\frac{y \sqrt{S c}}{2 \sqrt{t}}+\sqrt{b_{4} t}\right)
\end{array}\right] \\
& u_{21}(y, t)=\frac{e^{b_{4} t}}{2}\left[\begin{array}{c}
e^{-y \sqrt{\operatorname{Pr}\left(b_{4}+b_{1}\right)}} \operatorname{erfc}\left(\frac{y \sqrt{\operatorname{Pr}}}{2 \sqrt{t}}-\sqrt{\left(b_{4}+b_{1}\right) t}\right)+ \\
e^{y \sqrt{\operatorname{Pr}\left(b_{4}+b_{1}\right)}} \operatorname{erfc}\left(\frac{y \sqrt{\operatorname{Pr}}}{2 \sqrt{t}}+\sqrt{\left(b_{4}+b_{1}\right) t}\right)
\end{array}\right]
\end{aligned}
$$

To obtain the expressions from $\mathrm{u}_{11}$ to $\mathrm{u}_{21}$ the MATLAB software is utilized and the same software is used to draw the graphical presentations.

The shear stress at the plate is given by 


$$
\begin{aligned}
& \tau=-\left(\frac{\partial u}{\partial y}\right)_{y=0}=\frac{1}{\sqrt{\pi t}} e^{-A_{1} t}+\sqrt{A_{1}}\left(1-\operatorname{erfc}\left(\sqrt{A_{1} t}\right)\right) \\
& -A_{7} e^{a t}\left[\sqrt{\frac{1}{\pi t}} e^{-\left(a+A_{1}\right) t}+\sqrt{\left(a+A_{1}\right)}\left(1-\operatorname{erfc}\left(\sqrt{\left(a+A_{1}\right) t}\right)\right)\right] \\
& +A_{7} e^{b_{8} t}\left[\sqrt{\frac{1}{\pi t}} e^{-\left(b_{8}+A_{1}\right) t}+\sqrt{\left(b_{8}+A_{1}\right)}\left(1-\operatorname{erfc}\left(\sqrt{\left(b_{8}+A_{1}\right) t}\right)\right)\right] \\
& -A_{8} e^{a t}\left[\sqrt{\frac{1}{\pi t}} e^{-\left(a+A_{1}\right) t}+\sqrt{\left(a+A_{1}\right)}\left(1-\operatorname{erfc}\left(\sqrt{\left(a+A_{1}\right) t}\right)\right)\right] \\
& -A_{9} e^{b_{4} t}\left[\sqrt{\frac{1}{\pi t}} e^{-\left(b_{4}+A_{1}\right) t}+\sqrt{\left(b_{4}+A_{1}\right)}\left(1-\operatorname{erfc}\left(\sqrt{\left(b_{4}+A_{1}\right) t}\right)\right)\right. \\
& +A_{9} e^{b_{9} t}\left[\sqrt{\frac{1}{\pi t}} e^{-\left(b_{9}+A_{1}\right) t}+\sqrt{\left(b_{9}+A_{1}\right)}\left(1-\operatorname{erfc}\left(\sqrt{\left(b_{9}+A_{1}\right) t}\right)\right)\right] \\
& -A_{10} e^{b_{4} t}\left[\sqrt{\frac{1}{\pi t}} e^{-\left(b_{4}+A_{1}\right) t}+\sqrt{\left(b_{4}+A_{1}\right)}\left(1-\operatorname{erfc}\left(\sqrt{\left(b_{4}+A_{1}\right) t}\right)\right)\right] \\
& +A_{10} e^{b_{8} t}\left[\sqrt{\frac{1}{\pi t}} e^{-\left(b_{8}+A_{1}\right) t}+\sqrt{\left(b_{8}+A_{1}\right)}\left(1-\operatorname{erfc}\left(\sqrt{\left(b_{8}+A_{1}\right) t}\right)\right)\right] \\
& +A_{7} e^{a t}\left[\sqrt{\frac{\operatorname{Pr}}{\pi t}} e^{-\left(a+b_{1}\right) t}+\sqrt{\operatorname{Pr}\left(a+b_{1}\right)}\left(1-\operatorname{erfc}\left(\sqrt{\left(a+b_{1}\right) t}\right)\right)\right] \\
& -A_{7} e^{b_{8} t}\left[\sqrt{\frac{\operatorname{Pr}}{\pi t}} e^{-\left(b_{8}+b_{1}\right) t}+\sqrt{\operatorname{Pr}\left(b_{8}+b_{1}\right)}\left(1-\operatorname{erfc}\left(\sqrt{\left(b_{8}+b_{1}\right) t}\right)\right)\right] \\
& +A_{8} e^{a t}\left[\sqrt{\frac{S c}{\pi t}} e^{-a t}+\sqrt{a S c}(1-\operatorname{erfc}(\sqrt{a t}))\right] \\
& -A_{8} e^{b_{9} t}\left[\sqrt{\frac{S c}{\pi t}} e^{-b_{9} t}+\sqrt{S c b_{9}}\left(1-\operatorname{erfc}\left(\sqrt{b_{9} t}\right)\right)\right] \\
& +A_{9} e^{b_{4} t}\left[\sqrt{\frac{S c}{\pi t}} e^{-b_{4} t}+\sqrt{b S c}(1-\operatorname{erfc}(\sqrt{a t}))\right] \\
& -A_{9} e^{b_{9} t}\left[\sqrt{\frac{S c}{\pi t}} e^{-b_{9} t}+\sqrt{S c b_{9}}\left(1-\operatorname{erfc}\left(\sqrt{b_{9} t}\right)\right)\right] \\
& +A_{10} e^{b_{4} t}\left[\sqrt{\frac{\operatorname{Pr}}{\pi t}} e^{-\left(b_{4}+b_{1}\right) t}+\sqrt{\operatorname{Pr}\left(b_{4}+b_{1}\right)}\left(1-\operatorname{erfc}\left(\sqrt{\left(b_{4}+b_{1}\right) t}\right)\right)\right] \\
& -A_{10} e^{b_{8} t}\left[\sqrt{\frac{\operatorname{Pr}}{\pi t}} e^{-\left(b_{8}+b_{1}\right) t}+\sqrt{\operatorname{Pr}\left(b_{8}+b_{1}\right)}\left(1-\operatorname{erfc}\left(\sqrt{\left(b_{8}+b_{1}\right) t}\right)\right)\right] \\
& N u=-\left(\frac{\partial \theta}{\partial y}\right)_{y=0}=e^{a t}\left[\sqrt{\frac{\operatorname{Pr}}{\pi t}} e^{-\left(a+b_{1}\right) t}+\sqrt{\operatorname{Pr}\left(a+b_{1}\right)}\left(1-\operatorname{erfc}\left(\sqrt{\left(a+b_{1}\right) t}\right)\right)\right]
\end{aligned}
$$




$$
\begin{aligned}
& S h=-\left(\frac{\partial C}{\partial y}\right)_{y=0}=\left(1+b_{6}\right) e^{a t}\left[\sqrt{\frac{S c}{\pi t}} e^{-a t}+\sqrt{a S c}(1-\operatorname{erfc}(\sqrt{a t}))\right] \\
& +b_{7} e^{b_{4} t}\left[\sqrt{\frac{S c}{\pi t}} e^{-b_{4} t}+\sqrt{b S c}(1-\operatorname{erfc}(\sqrt{a t}))\right] \\
& -b_{6} e^{a t}\left[\sqrt{\frac{\operatorname{Pr}}{\pi t}} e^{-\left(a+b_{1}\right) t}+\sqrt{\operatorname{Pr}\left(a+b_{1}\right)}\left(1-\operatorname{erfc}\left(\sqrt{\left(a+b_{1}\right) t}\right)\right)\right] \\
& -b_{7} e^{b_{4} t}\left[\sqrt{\frac{\operatorname{Pr}}{\pi t}} e^{-\left(b_{4}+b_{1}\right) t}+\sqrt{\operatorname{Pr}\left(b_{4}+b_{1}\right)}\left(1-\operatorname{erfc}\left(\sqrt{\left(b_{4}+b_{1}\right) t}\right)\right)\right]
\end{aligned}
$$

\section{Results and discussion}

To analyze the properties of the flow pattern under the effects of different parameters encountered in the governing equations, graphical representations are employed as per the boundary conditions considered.

The variations in flow velocity are depicted in Figs. 2-5. Fig. 2 \& Fig. 3 display the influence of Grashof numbers for heat transfer and mass transfer on velocity distribution respectively. It reveals that the velocity get boosting for increasing values of both the numbers. This happens due to the fact that the buoyancy force which acts on the fluid particles due to gravitational forces. As a result the fluid velocity enhances. In Fig. 4, velocity profiles are displayed with the effect of magnetic parameter. The values of magnetic parameter are taken relevant to the expressions and remaining physical parameters to check the variations in velocity. It is seen that velocity gets reduced by the increase of magnetic parameter. Similar approach is noticed by Raju et al. [1]. Fig. 5 demonstrates that the velocity grows with an augment in porosity parameter. This happens due to the fact that rising values of $K$ leads to reduce the drag force which enables the fluid considerably to move fast. Also the velocity attains uniformity for some maximum value of porosity parameter. This is due to the natural phenomena that higher values of porosity parameter gives free movement for velocity without any retard.

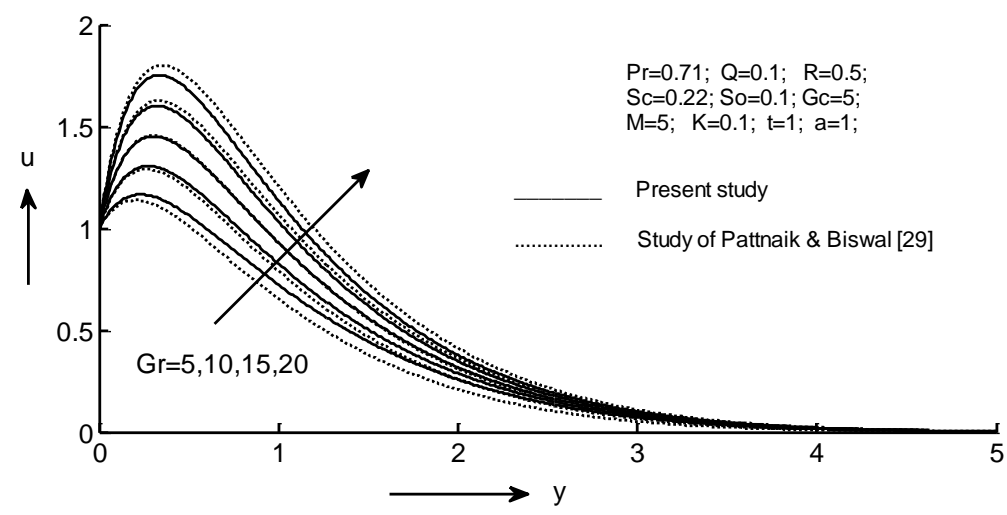

Fig. 2. Comparison of present study with the existing literature the effect of Grashof number on velocity distribution in the absence of thermal radiation and Soret effect. 


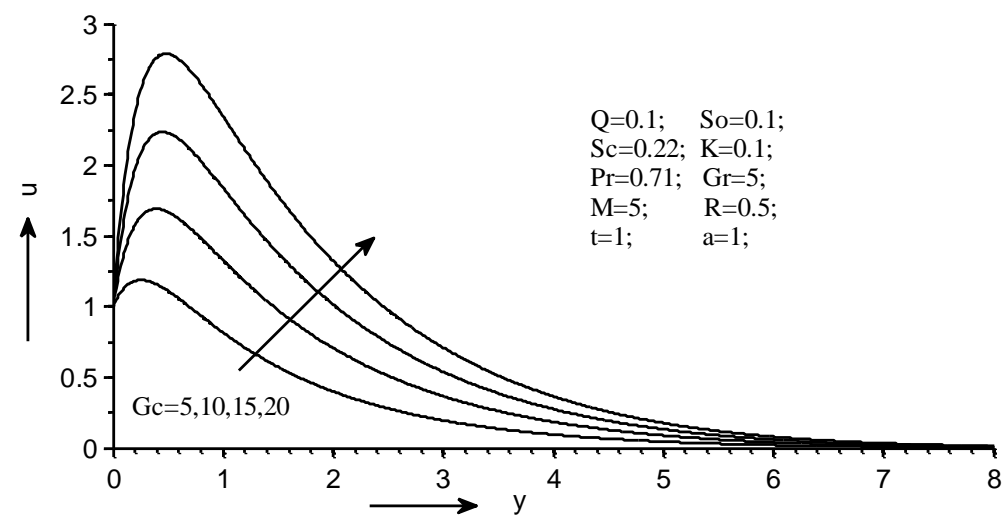

Fig. 3. Effect of modified Grashof number on velocity distribution

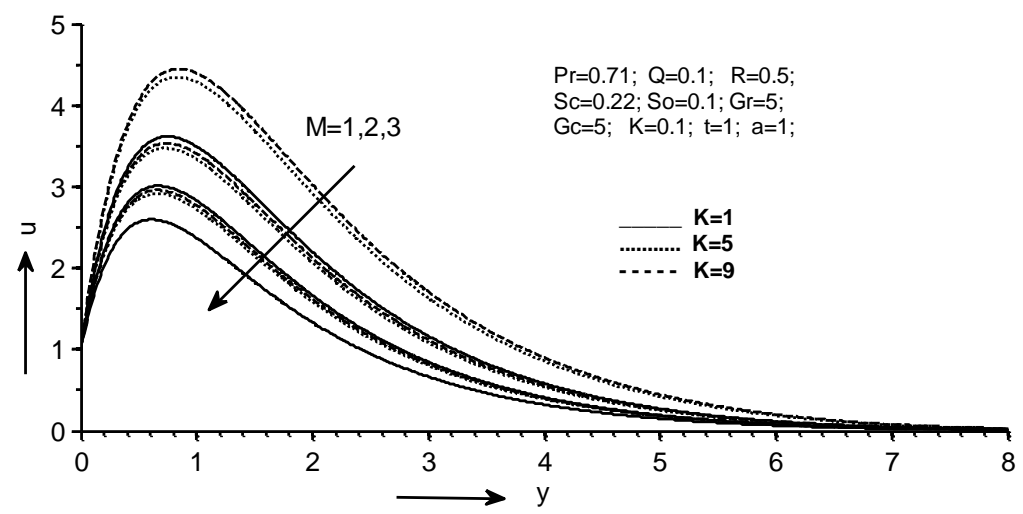

Fig. 4. Effect of Magnetic parameter on velocity distribution with varying porosity parameter

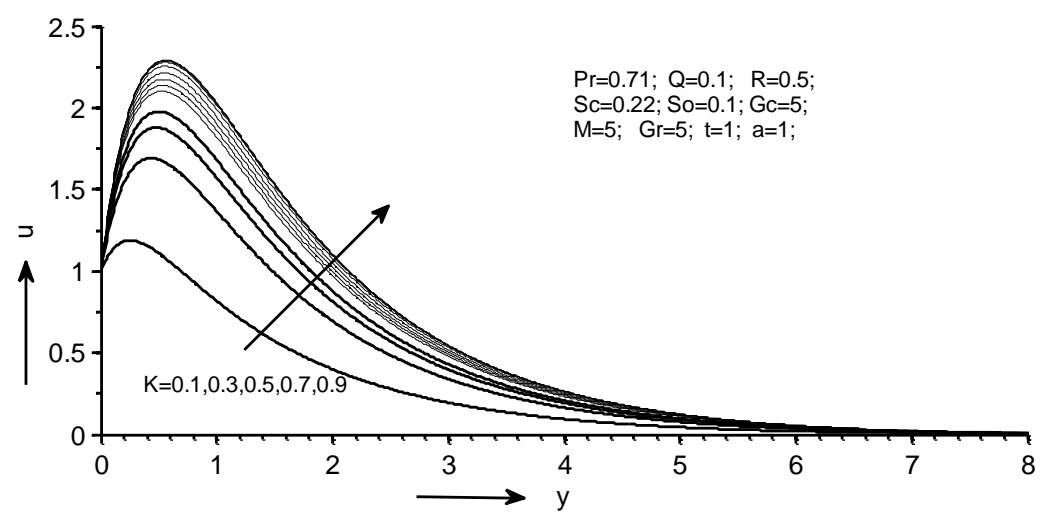

Fig. 5. Effect of porosity parameter on velocity distribution

Figs. 6(a) and 6(b) illustrate the effect of Prandtl number on the fluid velocity and temperature. It is shown that rising values of Prandtl number leads to decrease the velocity and 
temperature. $\operatorname{Pr}=0.71$ is to determine the thermal conductivity of air. Pr values around 3 to 7 are for analysis on base fluid water. When Prandtl number is less than one the thermal diffusivity dominates the behavior. When Prandtl number is greater than one the molecular diffusivity dominates the behavior. Figs. 7(a), 7(b) and 7(c) point up the effect of heat source/sink on the velocity, temperature and concentration distributions. The velocity as well as temperature gets improved in the presence of heat source whereas they become thinner with heat sink impact. The fluid concentration diminishes with ascending values of heat source parameter and an opposite trend is created with heat sink influence. Figs. 8(a), 8(b) and 8(c) pointed the changes in velocity, temperature and concentration with the effect of time. As time elapses, enhancement in all these parameters is found. The small values of time with difference 0.2 is taken in order to analyze the variations very closely

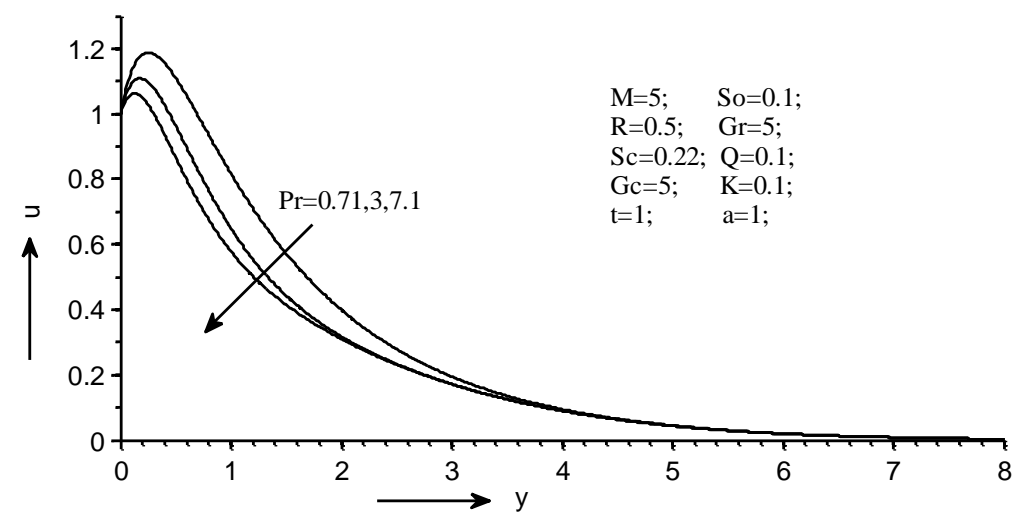

Fig. 6(a). Effect of Prandtl number on velocity distribution

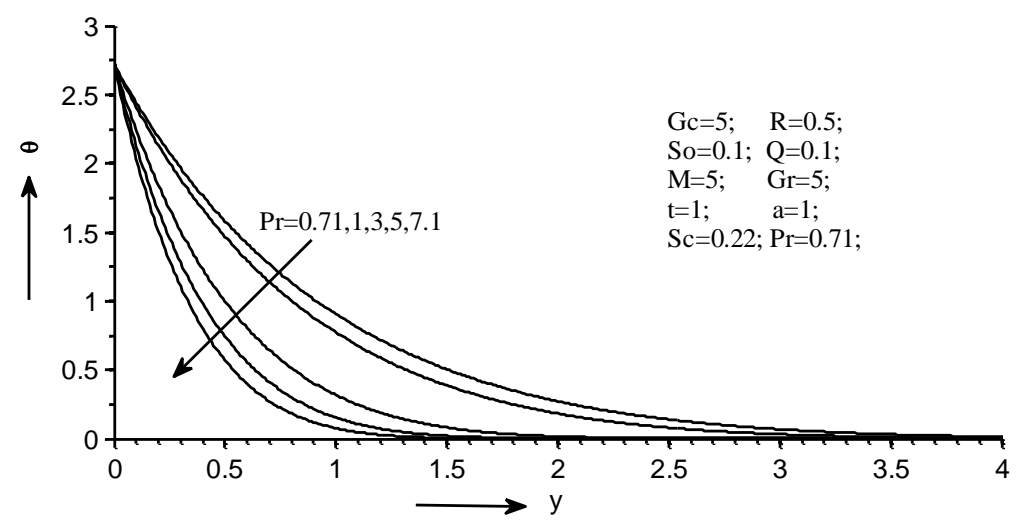

Fig. 6(b). Effect of Prandtl number on temperature distribution 


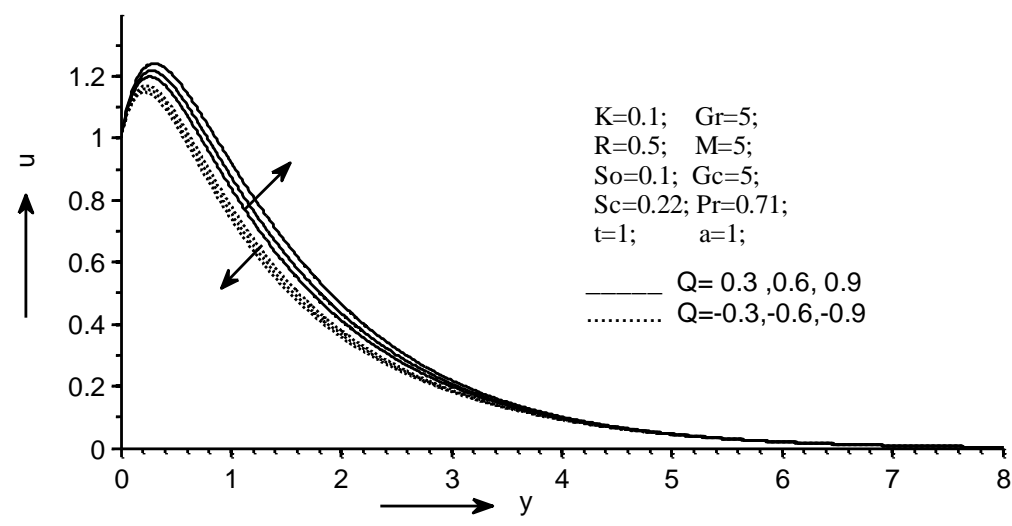

Fig. 7(a). Effect of heat generation/absorption on velocity distribution

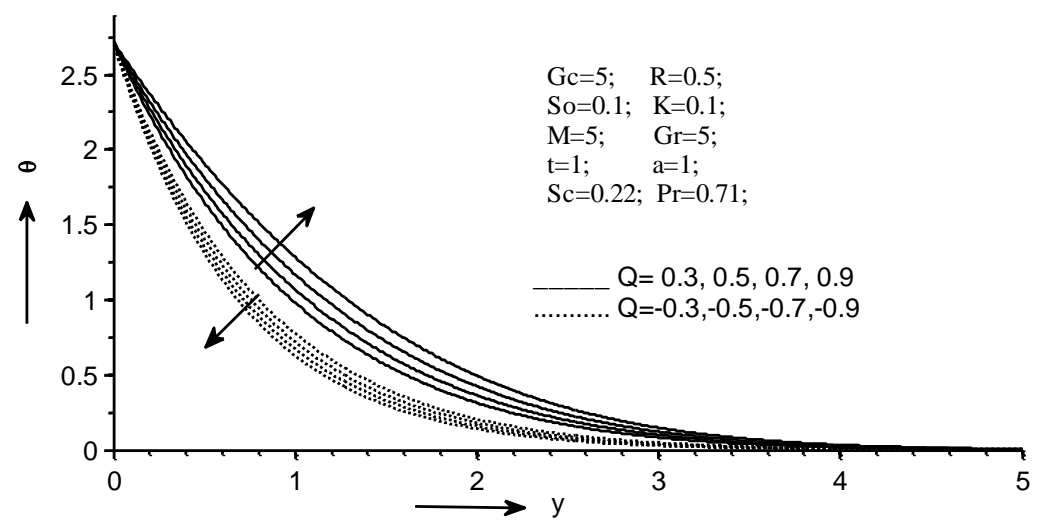

Fig. 7(b). Effect of heat generation/absorption on temperature distribution

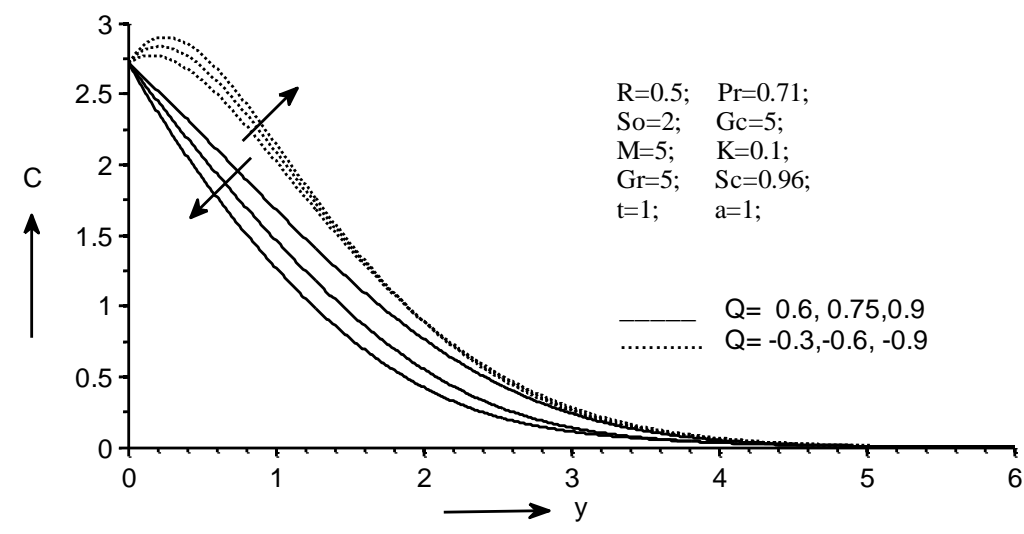

Fig. 7(c). Effect of heat generation/absorption on fluid concentration 


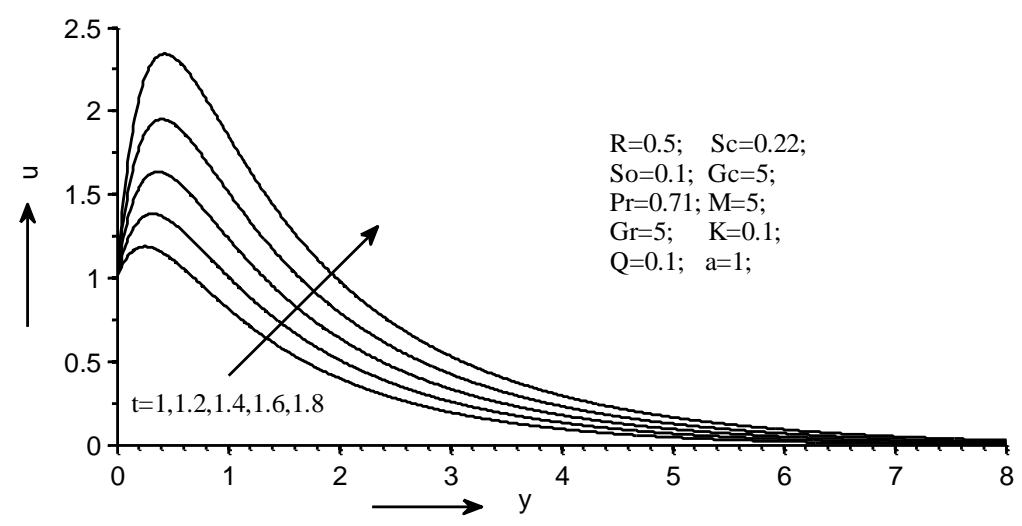

Fig. 8(a). Effect of time on velocity distribution

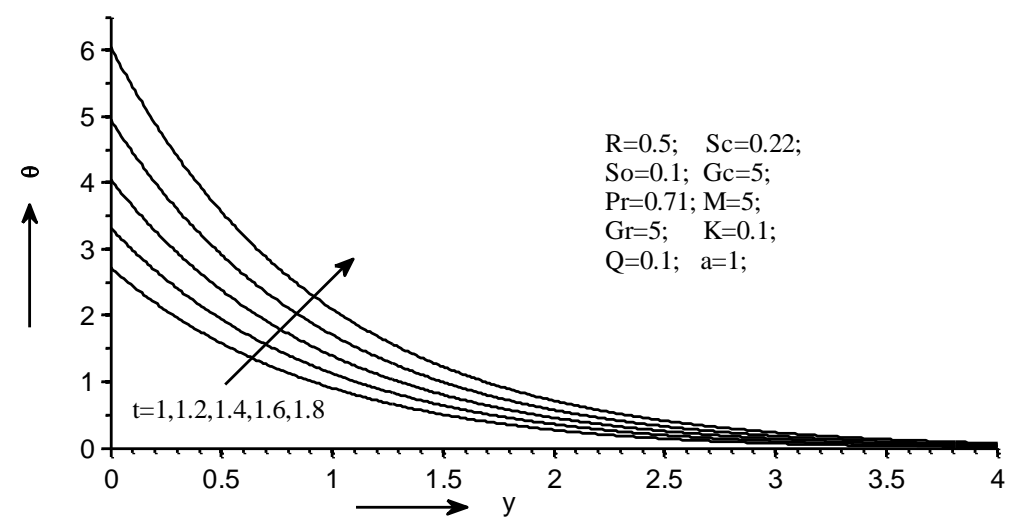

Fig. 8(b). Effect of time on temperature distribution

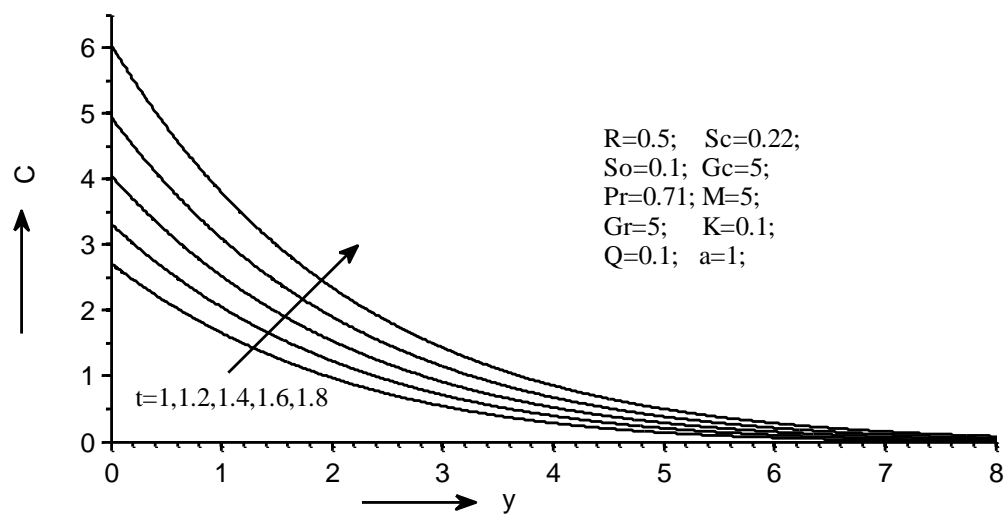

Fig. 8(c). Effect of time on fluid concentration

The variations in the velocity and concentration distributions under the influence of Schmidt number is displayed in Figs. 9(a) and 9(b). The Schmidt number is the fraction of the force diffusivity to the species (mass) diffusivity. Both of them become thinner for mounting values of 
this number. When heat \& mass transfer transpire at once in a affecting fluid, the mass flux generated by temperature gradients is termed as thermal-diffusion (Soret effect). The values of Schmidt number are taken as $0.22,0.60,0.78$ and 0.96 for hydrogen, ammonia, oxygen and methane respectively. The velocity as well as species concentration improves for expanding values of this number. This is evident from Figs. 10(a) and 10(b). This takes place due to the dispersion of heated molecules which reduces the density and results in growing the velocity distribution as well as concentration. Figs. 11(a) and 11(b) demonstrate the cause of thermal emission on fluid temperature and concentration. The temperature falls down for increasing values of radiation parameter whereas a repeal tendency is observed in the case of concentration.

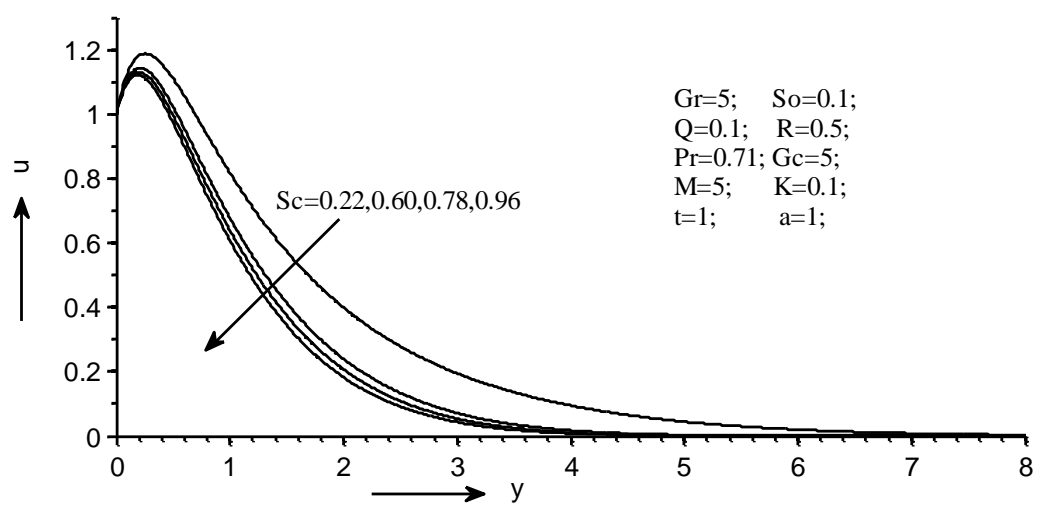

Fig. 9(a). Effect of Schmidt number on velocity distribution

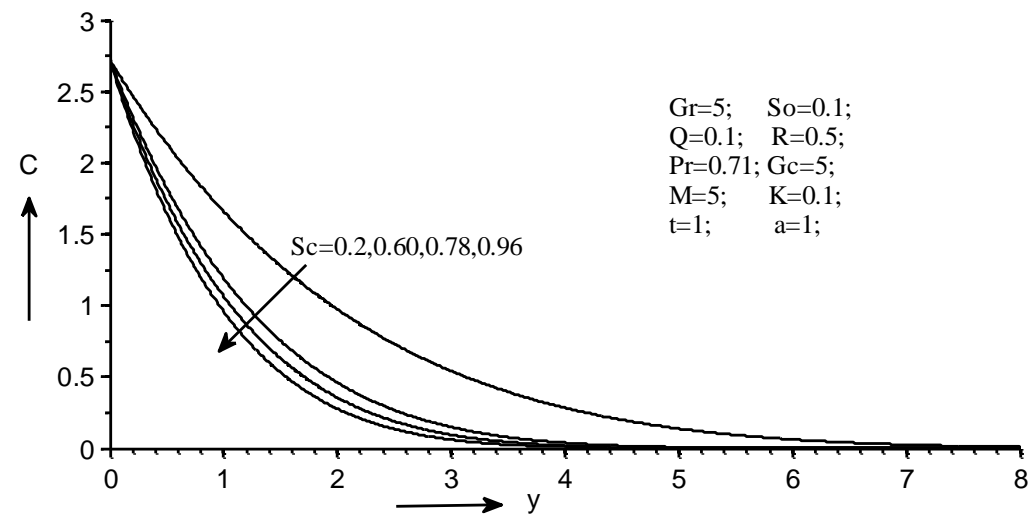

Fig. 9(b). Effect of Schmidt number on fluid concentration 


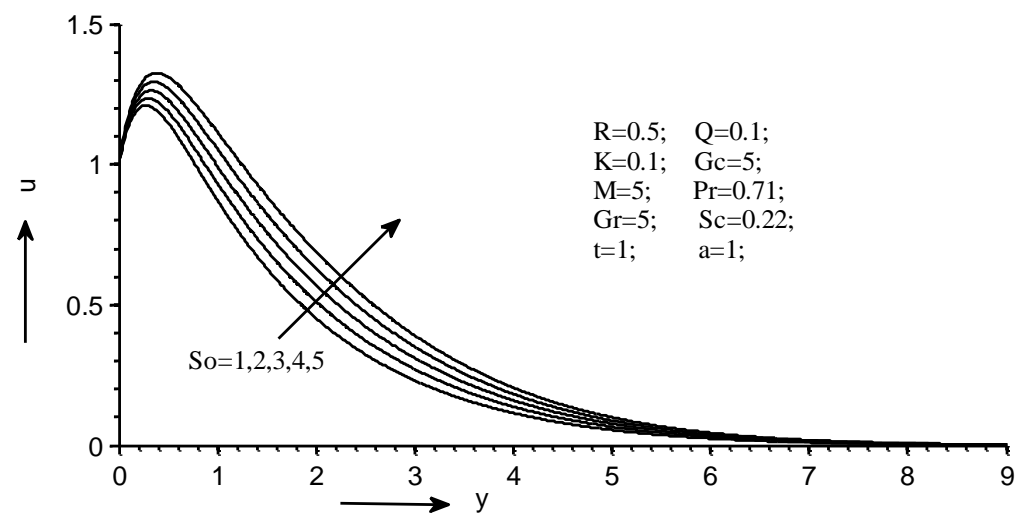

Fig. 10(a). Effect of Soret number on velocity distribution

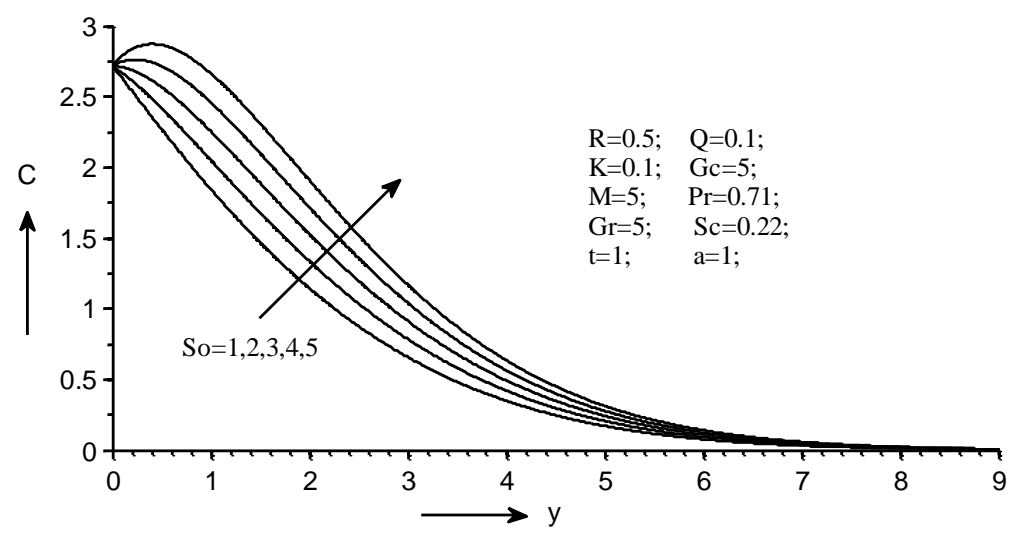

Fig. 10(b). Effect of Soret number on fluid concentration

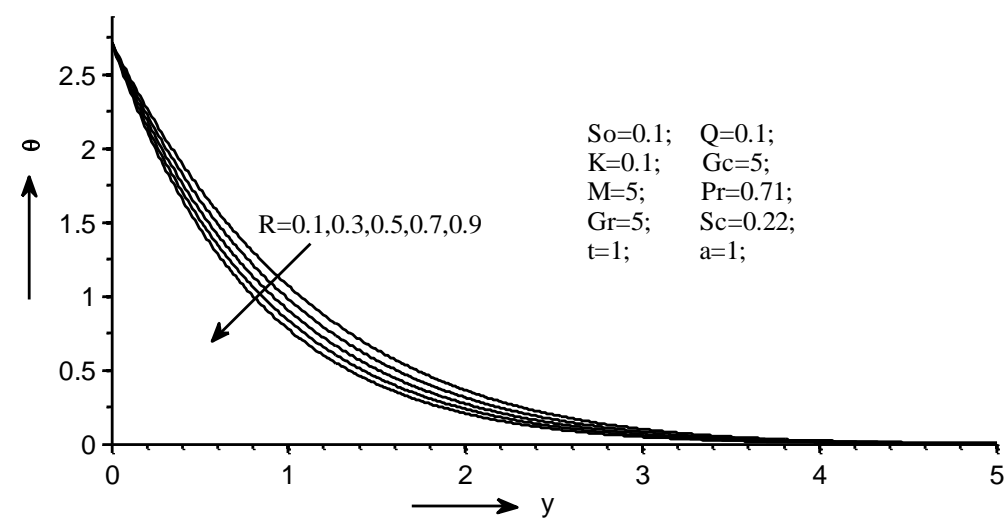

Fig. 11(a). Effect of radiation parameter on temperature distribution 


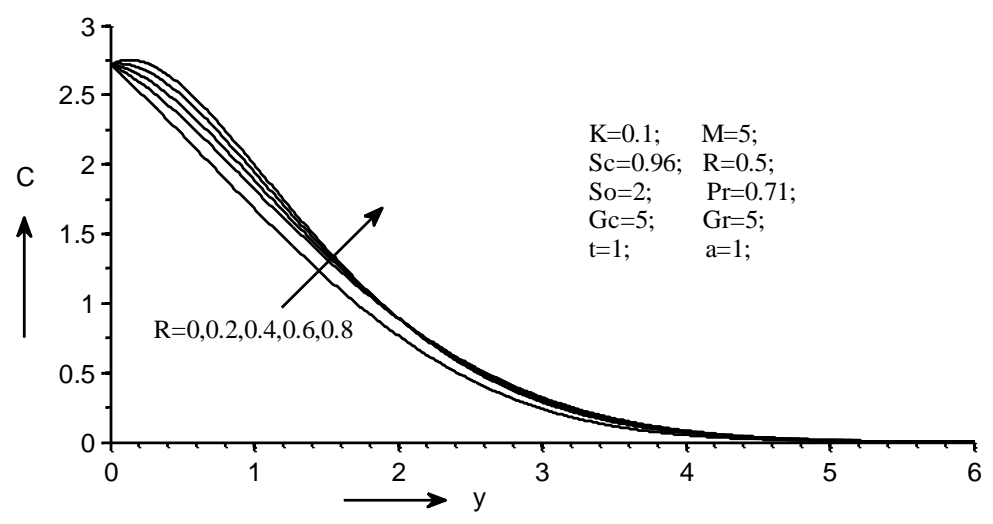

Fig. 11(b). Effect of radiation parameter on fluid concentration

In accumulation to these properties we examined the impact of thermal diffusion on velocity and concentration by changing the values of radiation. From Figs. 12(a) and 12(b) it is noticed that the velocity as well as concentration increases by enhancing the Soret number and also for improved values of radiation. We concluded that the impact of radiation is more on concentration when compared to that of velocity of the flow.

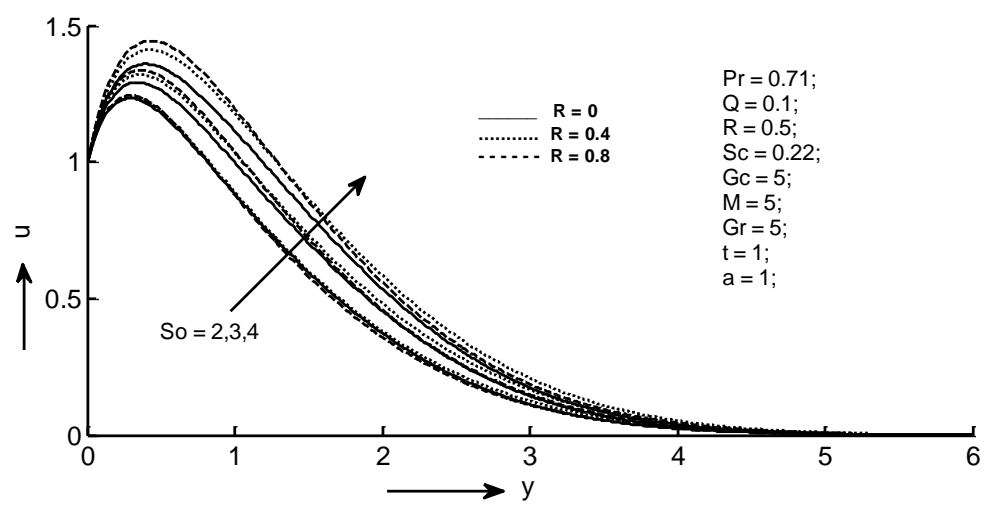

Fig. 12(a). Effect of thermal diffusion on fluid velocity for varying values of radiation

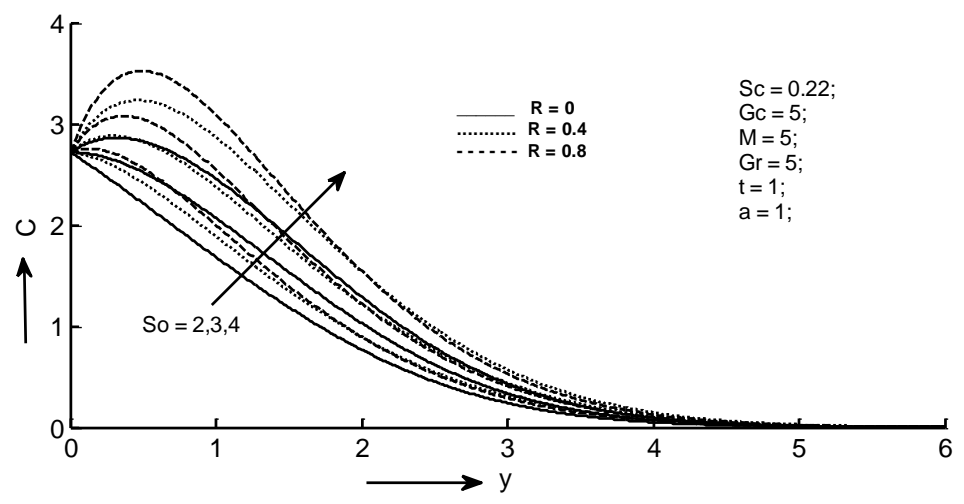

Fig. 12(b). Effect of thermal diffusion on fluid concentration for varying values of radiation 
We also extended the present work in analyzing the effects of special parameters on shear stress, rates of heat and mass transfer by using tabular values. Table 1 illustrates the variations in shear stress at the plate under the consequence of diverse parameters. Skin rubbing decreases for ascending values of Grashof number of heat transfer, Grashof number of mass transfer, porosity parameter and Soret number. The rising values of magnetic field parameter, Prandtl integer and Schmidt amount leads to increase the skin friction. Table 2 reveals that heat transmit rate enhances under the effect of Prandtl amount and radiation parameter but a repeal tendency is found under heat source existence. Sherwood number increases with the bigger values of Schmidt numeral and declining values of Soret number. This is evident from table 3.

\begin{tabular}{|c|c|c|c|c|c|c|c|}
\hline $\mathbf{G r}$ & $\mathbf{G c}$ & $\mathbf{M}$ & $\mathbf{P r}$ & $\mathbf{K}$ & $\mathbf{S c}$ & So & $\boldsymbol{\tau}$ \\
\hline $\mathbf{1}$ & 1 & 15 & 0.71 & 0.5 & 0.22 & 0.1 & $\mathbf{3 . 0 0 6 9}$ \\
$\mathbf{2}$ & 1 & 15 & 0.71 & 0.5 & 0.22 & 0.1 & $\mathbf{2 . 4 8 0 6}$ \\
$\mathbf{3}$ & 1 & 15 & 0.71 & 0.5 & 0.22 & 0.1 & $\mathbf{1 . 9 5 4 3}$ \\
$\mathbf{4}$ & 1 & 15 & 0.71 & 0.5 & 0.22 & 0.1 & $\mathbf{1 . 4 2 8 0}$ \\
\hline 1 & $\mathbf{1}$ & 15 & 0.71 & 0.5 & 0.22 & 0.1 & $\mathbf{3 . 0 0 6 9}$ \\
1 & $\mathbf{2}$ & 15 & 0.71 & 0.5 & 0.22 & 0.1 & $\mathbf{2 . 4 1 7 0}$ \\
1 & $\mathbf{3}$ & 15 & 0.71 & 0.5 & 0.22 & 0.1 & $\mathbf{1 . 8 2 7 1}$ \\
1 & $\mathbf{4}$ & 15 & 0.71 & 0.5 & 0.22 & 0.1 & $\mathbf{1 . 2 3 7 2}$ \\
\hline 1 & 1 & $\mathbf{5}$ & 0.71 & 0.5 & 0.22 & 0.1 & $\mathbf{1 . 0 6 0 3}$ \\
1 & 1 & $\mathbf{1 0}$ & 0.71 & 0.5 & 0.22 & 0.1 & $\mathbf{2 . 1 7 7 1}$ \\
1 & 1 & $\mathbf{1 5}$ & 0.71 & 0.5 & 0.22 & 0.1 & $\mathbf{3 . 0 0 6 9}$ \\
1 & 1 & $\mathbf{2 0}$ & 0.71 & 0.5 & 0.22 & 0.1 & $\mathbf{3 . 6 8 9 1}$ \\
\hline 1 & 1 & 15 & $\mathbf{0 . 7 1}$ & 0.5 & 0.22 & 0.1 & $\mathbf{3 . 0 0 6 9}$ \\
1 & 1 & 15 & $\mathbf{3}$ & 0.5 & 0.22 & 0.1 & $\mathbf{3 . 0 9 4 9}$ \\
1 & 1 & 15 & $\mathbf{5}$ & 0.5 & 0.22 & 0.1 & $\mathbf{3 . 1 2 9 7}$ \\
1 & 1 & 15 & $\mathbf{7 . 1}$ & 0.5 & 0.22 & 0.1 & $\mathbf{3 . 1 5 5 6}$ \\
\hline 1 & 1 & 15 & 0.71 & $\mathbf{0 . 1}$ & 0.22 & 0.1 & $\mathbf{4 . 0 5 2 1}$ \\
1 & 1 & 15 & 0.71 & $\mathbf{0 . 3}$ & 0.22 & 0.1 & $\mathbf{3 . 2 0 0 2}$ \\
1 & 1 & 15 & 0.71 & $\mathbf{0 . 5}$ & 0.22 & 0.1 & $\mathbf{3 . 0 0 6 9}$ \\
1 & 1 & 15 & 0.71 & $\mathbf{0 . 7}$ & 0.22 & 0.1 & $\mathbf{2 . 9 2 1 0}$ \\
\hline 1 & 1 & 15 & 0.71 & 0.5 & $\mathbf{0 . 2 2}$ & 0.1 & $\mathbf{3 . 0 0 6 9}$ \\
1 & 1 & 15 & 0.71 & 0.5 & $\mathbf{0 . 6 0}$ & 0.1 & $\mathbf{3 . 0 3 1 5}$ \\
1 & 1 & 15 & 0.71 & 0.5 & $\mathbf{0 . 7 8}$ & 0.1 & $\mathbf{3 . 0 4 9 9}$ \\
1 & 1 & 15 & 0.71 & 0.5 & $\mathbf{0 . 9 6}$ & 0.1 & $\mathbf{3 . 0 5 1 1}$ \\
\hline 1 & 1 & 15 & 0.71 & 0.5 & 0.22 & $\mathbf{1}$ & $\mathbf{2 . 9 9 1 6}$ \\
1 & 1 & 15 & 0.71 & 0.5 & 0.22 & $\mathbf{2}$ & $\mathbf{2 . 9 7 4 6}$ \\
1 & 1 & 15 & 0.71 & 0.5 & 0.22 & $\mathbf{3}$ & $\mathbf{2 . 9 5 7 6}$ \\
1 & 1 & 15 & 0.71 & 0.5 & 0.22 & $\mathbf{4}$ & $\mathbf{2 . 9 4 0 7}$ \\
\hline
\end{tabular}

Table 1. Variations in shear stress at the plate under the effect of different parameters 


\begin{tabular}{|c|c|c|c|}
\hline $\mathbf{P r}$ & $\mathbf{Q}$ & $\mathbf{R}$ & $\mathrm{Nu}$ \\
\hline $\mathbf{0 . 7 1}$ & 0.1 & 0.5 & $\mathbf{2 . 9 1 4 0}$ \\
$\mathbf{1}$ & 0.1 & 0.5 & $\mathbf{3 . 2 9 1 3}$ \\
$\mathbf{3}$ & 0.1 & 0.5 & $\mathbf{5 . 2 0 4 9}$ \\
7.1 & 0.1 & 0.5 & $\mathbf{7 . 7 7 7 8}$ \\
\hline 0.71 & $\mathbf{0 . 1}$ & 0.5 & $\mathbf{2 . 9 1 4 0}$ \\
0.71 & $\mathbf{0 . 3}$ & 0.5 & $\mathbf{2 . 6 6 8 2}$ \\
0.71 & $\mathbf{0 . 5}$ & 0.5 & $\mathbf{2 . 4 0 5 6}$ \\
0.71 & $\mathbf{0 . 7}$ & 0.5 & $\mathbf{2 . 1 2 3 5}$ \\
\hline 0.71 & 0.1 & $\mathbf{0 . 1}$ & $\mathbf{2 . 4 0 5 6}$ \\
0.71 & 0.1 & $\mathbf{0 . 3}$ & $\mathbf{2 . 6 6 8 2}$ \\
0.71 & 0.1 & $\mathbf{0 . 5}$ & $\mathbf{2 . 9 1 4 0}$ \\
0.71 & 0.1 & $\mathbf{0 . 7}$ & $\mathbf{3 . 1 4 5 1}$ \\
\hline
\end{tabular}

Table 2. Effect of Prandtl number and radiation parameter on rate of heat transfer

\begin{tabular}{|c|c|c|}
\hline Sc & So & Sh \\
\hline $\mathbf{0 . 2 2}$ & 0.1 & $\mathbf{1 . 2 9 5 8}$ \\
$\mathbf{0 . 6 0}$ & 0.1 & $\mathbf{2 . 1 6 2 3}$ \\
$\mathbf{0 . 7 8}$ & 0.1 & $\mathbf{2 . 3 9 9 8}$ \\
$\mathbf{0 . 9 6}$ & 0.1 & $\mathbf{2 . 6 5 4 9}$ \\
\hline 0.22 & $\mathbf{0 . 5}$ & $\mathbf{1 . 1 2 2 9}$ \\
0.22 & $\mathbf{1}$ & $\mathbf{0 . 9 0 6 7}$ \\
0.22 & $\mathbf{1 . 5}$ & $\mathbf{0 . 6 9 0 5}$ \\
0.22 & $\mathbf{2}$ & $\mathbf{0 . 4 7 4 3}$ \\
\hline
\end{tabular}

Table 3. Dependence between Sherwood, Schmidt and Soret number

\section{Comparison /Validation of results:}

In order to check the accuracy of the method of solution a comparison has made between the present study and the study of Pattnaik and Biswal [29] (see Fig 2) by considering the behavior of the velocity under the effect of Grashof number in the absence of thermal radiation and Soret effect. We noticed that the two velocity distributions are almost identical as the velocity increases for rising values of Grashof number. A good agreement is found in the comparison of the present results with that of Pattnaik and Biswal [29] and the accuracy of the method is confirmed.

\section{Conclusion:}

The present effort is committed to learn the properties of changeable temperature and concentration effects on MHD free convective radiating fluid past a leaky plate in the occurrence of thermal diffusion with heat generation/absorption. The consideration of thermal radiation and Soret effects along with variable temperature and concentration is chosen as novelty based on its importance in various fields of research. Also many researchers studied on thermal radiation and Soret effects with constant temperature and concentration. In view of this literature variable temperature and concentration is considered and also analysis is done with variety of graphical presentations. The main points observed in this study can be briefed as follows: 
- Fluid velocity grows up with ascending values of porosity parameter, Grashof number, molecular Grashof number and decreasing values of magnetic parameter.

- In the incidence of heat source the fluid velocity and temperature increases whereas the concentration decreases. The velocity and temperature falls down in the presence of heat sink but a reverse trend is found in the species concentration.

- Increasing values of Soret number serves to enhance the velocity and species concentration but an opposite nature is seen in the case of Schmidt number.

- The impact of radiation is more on concentration when compared to that of velocity of the flow for varying values of Soret number.

- Rising values of magnetic factor, Prandtl numeral and Schmidt quantity leads to increase the skin friction.

- The rate of heat transfer enhances under the effect of Prandtl number and radiation parameter but a reverse trend is seen in the case of heat source.

\section{References:}

Ahmed S and Kalita K (2013). Magnetohydrodynamic transient flow through a porous medium bounded by a hot vertical plate in the presence of radiation: a theoretical analysis, Jou. of Engg. Physics and Thermophysics, 86, 30-39.

Awais M, Hayat T, Aamir Ali and Irum S (2016). Velocity, thermal and concentration slip effects on a magneto-hydrodynamic nanofluid flow, Alexandria Engineering Journal, 55, 21072114 .

Azad Hussain, Anwar Ullah (2016). Boundary layer flow of a Walter's B fluid due to a stretching cylinder with temperature dependent viscosity, Alexandria Engineering Journal, 55, 30733080.

Chamkha A J, El-Amin M F and Aly A M (2011). Unsteady double diffusive natural convective MHD flow along a vertical cylinder in the presence of chemical reaction, thermal radiation and Soret and Dufour effects, Jou. of Naval Arch. and Marine Engg., 8, 25-36.

Chamkha A J, Mansour M A and Aly A M (2011). Unsteady MHD free convective heat and mass transfer from a vertical porous plate with hall current, thermal radiation and chemical reaction effects, Int. Jou. for numerical methods in fluids, 65, 432-447.

Das M, Mahato R, Nandkeolyar R (2015). Newtonian heating effect on unsteady hydromagnetic Casson fluid flow past a flat plate with heat and mass transfer, Alexandria Engineering Journal, 54, 871-879.

Das S, Mandal C and Jana R N (2012). Radiation effects on unsteady free convection flow past a vertical plate with Newtonian heating, Int. Jou. Comput. Appl., 41, 36-41.

Hayat T, Qasim M (2010). Influence of thermal radiation and Joule heating on MHD flow of a Maxwell fluid in the presence of thermophoresis, Int. Jou. heat \& mass transfer, 53, 47804788.

Hayat T, Shehzad S A, Ashraf M B, Alsaedi A (2013). Magnetohydrodynamic mixed convection flow of thixotropic fluid with thermophoresis and Joule heating, J. Thermophys. Heat Transfer, 27, 733-740.

Hossain M A, Khanafer K and Vafai K (2001). The effect of radiation on free convection flow of fluid with variable viscosity from a porous vertical plate, Int. Jou. of Thermal Sciences, 40(2), 115-124. 
Kandasamy R, Periasamy K, Prabhu K K S (2005). Effects of chemical reaction, heat and mass transfer along a wedge with heat source and concentration in the presence of suction or injection, Int. Jou. Heat and mass transfer, 48(7), 1388-1394.

Kim Y J (2001). Unsteady MHD convection flow of polar fluids past a vertical moving porous plate in a porous medium, Int. Jou. of Heat and Mass Transfer, 44, 2791-2799.

Makinde O D, Khan W A, Khan Z H (2013). Buoyancy effects on MHD stagnation point flow and heat transfer of a nanofluid past a convectively heated stretching/shrinking sheet, Int. Jou. heat mass transfer, 62, 526-533.

Murthy P V S N and El-Amin M F (2011). Thermo-diffusion effect on free convection heat and mass transfer in a thermally stratified non-darcy porous media, The Open Transport Phenomenon Journal, 3, 49-55.

Muthucumaraswamy R, Sundarraj M and Subhramanian V (2009). Unsteady flow past an accelerated infinite vertical plate with variable temperature and uniform mass diffusion, Int. Jou. Appl. Math. Mech, 5, 51-6.

Muthucumarswamy R and Kumar GS (2004). Heat and mass transfer effects on moving vertical plate in the presence of thermal radiation, Theoret. Appl. Mach., 31(1), 35-46.

Narahari M and Nayan MY (2011). Free convection flow past an impulsively started infinite vertical plate with Newtonian heating in the presence of thermal radiation and mass diffusion, Turk. Jou. Eng. Environ. Sci., 35, 187-198.

Narayana M, Khidir AA, Sibanda P and Murthy PVSN (2013). Soret effect on the natural convection from a vertical plate in a thermally stratified porous medium saturated with nonNewtonian liquid, Jou. of Heat Transfer, 135-03251-1.

Pattnaik PK and Biswal T (2015). Analytical solution of MHD free convective flow through porous media with time dependent temperature and concentration, Walailak Jou. Sci \& Tech, 12(9), 749-762.

Raju MC, Varma SVK, Reddy NA (2011). MHD thermal diffusion natural convection flow between heated inclined plates in porous medium, Jou. on Future Engineering and Tech., 6(2), 45-48.

Raptis A (1986). Flow through a porous medium in the presence of magnetic field, Int. Jou. Energy Res., 10, 97-101.

Reddy SH, Keshava Reddy E, Raju MC (2015). Unsteady MHD free convection flow of a Kuvshinski fluid past a vertical porous plate in the presence of chemical reaction and heat source/sink, Int. Jou. of Engg. Research in Africa, 14, 13-27.

Reddy TS, Raju MC and Varma SVK (2013). Unsteady MHD radiative and chemically reactive free convection flow near a moving vertical plate in porous medium, Jou. of Applied Fluid Mech., 6(3), 443-451.

Sandeep N, Reddy AVB, Sugunamma V (2012). Effect of radiation and chemical reaction on transient MHD free convective flow over a vertical plate through porous media, Chemical and process engg. Research, $2,1-9$.

Seddeek MA (2000). The effect of variable viscosity on hydromagnetic flow and heat transfer past a continuously moving porous boundary with radiation, Int. Com., Heat Mass Transfer, 27(7), 1037-1046.

Seth GS, Sarkar S, Hussain SM and Mahato GK (2015). Effects of hall current and rotation on hydromagnetic natural convection flow with heat and mass transfer of a heat absorbing fluid past an impulsively moving vertical plate with ramped temperature, Jou. Appl. Fluid Mech., 8(1), 159-171.

Sharma BK, Sharma PK and Chand T (2011). Effect of radiation on temperature distribution in three-dimensional Couette flow with heat source/sink, Int. Jou. of Applied Mechanics and Engg., 16(2), 531-542. 
Shibdas Dholey (2016). Free convection boundary layer flow of a viscous fluid above a hot horizontal semi-infinite flat plate with prescribed surface temperature, Alexandria Engineering Journal, 55, 2449-2461.

Srinivasacharya D and RamReddy C (2013). Cross-diffusion effects on mixed convection from an exponentially stretching surface in non-darcy porous medium, heat transfer- Asian Research, 42(2), 111-124.

Suneetha S, Reddy NB and Prasad VR (2008). Thermal radiation effects on MHD free convection flow past an impulsively started vertical plate with variable surface temperature and concentration, Jou. Naval Archit. Marine Eng., 2, 57-70.

Uwanta IJ and Sarki MN (2012). Heat and mass transfer with variable temperature and exponential mass diffusion, Int. Jou. Comput. Engg. Res., 2, 1487-94.

Vajravelu K, Kumar BVR (2004). Analytic and numerical solutions of coupled nonlinear system arising in three-dimensional rotating flow, Int. Jou. Non-Linear Mech., 39, 13-24. 